University of Nebraska - Lincoln

DigitalCommons@University of Nebraska - Lincoln

Biochemistry -- Faculty Publications

Biochemistry, Department of

1989

\title{
MULTIPLE ISOTOPE EFFECTS ON ENZYME-CATALYZED REACTIONS
}

Marion H. O'Leary

University of Nebraska - Lincoln

Follow this and additional works at: https://digitalcommons.unl.edu/biochemfacpub

Part of the Biochemistry Commons, Biotechnology Commons, and the Other Biochemistry, Biophysics, and Structural Biology Commons

O'Leary, Marion H., "MULTIPLE ISOTOPE EFFECTS ON ENZYME-CATALYZED REACTIONS" (1989).

Biochemistry -- Faculty Publications. 457.

https://digitalcommons.unl.edu/biochemfacpub/457

This Article is brought to you for free and open access by the Biochemistry, Department of at DigitalCommons@University of Nebraska - Lincoln. It has been accepted for inclusion in Biochemistry -- Faculty Publications by an authorized administrator of DigitalCommons@University of Nebraska - Lincoln. 


\title{
MULTIPLE ISOTOPE EFFECTS ON ENZYME-CATALYZED REACTIONS
}

\author{
Marion H. O'Leary
}

Department of Biochemistry, University of Nebraska-Lincoln, Lincoln, Nebraska 68583

\section{CONTENTS}

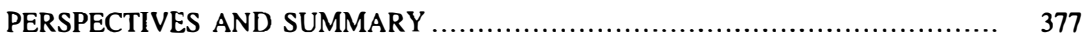

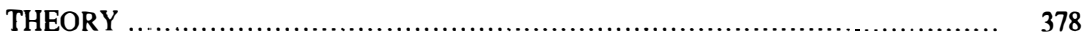

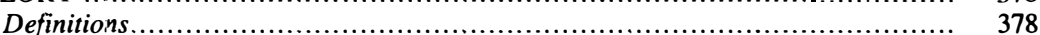

lsotope Effects and Enzyme Kinetics......................................................... 379

Quantitative Relationships.................................................. $\quad 380$

The Multiple Isotope Effect Method........................................................ 381

Numerical Solutions .............................................................. 382

The Rule of the Geometric Mean............................................ 382

ISOTOPE EFFECTS IN IRREVERSIBLE REACTIONS ........................... 383

Single-Step Reactions ........................................................ 383

Stepwise Mechanisms with Both Isotope-Sensitive Steps the Same..................... 386

Stepwise Mechanisms with Deuterium-Sensitive Step First .......................... 387

Stepwise Mechanisms with the ${ }^{13}$ C-Sensitive Step First ............................ 391

Stepwise Mechanisms with Two Isotope-Sensitive Steps........................... 391

REVERSIBLE REACTIONS .................................................... 392

APPLICATIONS USING SOLVENT ISOTOPE EFFECTS ......................... 394

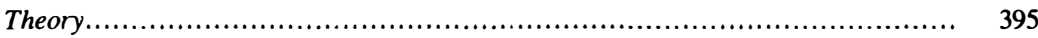

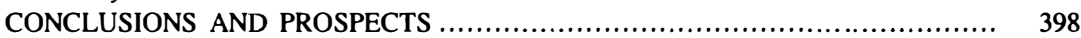

Precision and Uncertainty ...................................................... 399

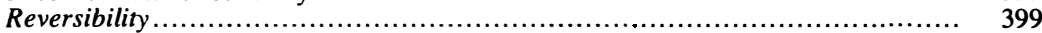

Theoretical Limitations ...................................................... 400

\section{PERSPECTIVES AND SUMMARY}

Isotope effects have long been popular as a method for studying mechanisms of chemical reactions $(1,2)$. Application of this method to enzymatic reactions has come slowly, in part because of the difficulty of making measure- 
ments of the necessary precision, and in part because of the difficulty of interpreting the variations in rate that occur in multistep reactions. The theory and practice of isotope effects has now reached the stage where a variety of interesting mechanistic studies are possible (3-9), including use of heavyatom isotope effects (5), application to multireactant enzymes $(9 \mathrm{a}, 10), \mathrm{pH}$ dependence of isotope effects $(11,12)$, proton inventory studies $(9)$, relative deuterium and tritium isotope effects (13), the remote label method (14), and the use of deuterium and tritium isotope effects for the study of tunneling ( $\mathrm{J}$. Klinman, personal communication).

Multiple isotope effects are a recent addition to this battery of techniques. They represent another example of the idea that isotopes are a minimal perturbation on a reaction system, and thus are particularly amenable to interpretation. In this case, an isotope effect for a substrate containing two isotopic substitutions is compared with the isotope effects for the corresponding singly substituted compounds. In various cases the two effects may be multiplicative (the simplest case), more than multiplicative, or less than multiplicative. The theory of this approach was developed by Cleland et al (15) and independently by Knowles et al $(16,17)$. The same technique has been used in studies of mechanisms of organic reactions $(18,19)$.

The multiple isotope effect technique can be used at two levels. First, it can be used qualitatively to distinguish between stepwise and concerted mechanisms, and in the case of stepwise mechanisms, it can be used to distinguish whether the step that gives rise to the hydrogen isotope effect precedes or follows the step that gives rise to the heavy-atom isotope effect. At a more quantitative level, the technique can be used to calculate isotope effects on individual steps and relative rates of various steps in an enzyme-catalyzed reaction. As we will see below, the latter approach requires a number of assumptions and cannot always be applied convincingly.

We first describe the nomenclature and theory of the multiple isotope effect method. Then we apply the method to reactions of gradually increasing complexity. In the last section, we consider limitations and prospects.

\section{THEORY}

The basic theory of the multiple isotope effect method has been described by Cleland et al (15).

\section{Definitions}

The nomenclature used for describing isotope effects is that of Northrop (20), in which isotope effects are designated by a leading superscript and the heavier isotope appears in the denominator of the rate or equilibrium ratio. Thus, ${ }^{13} k=k^{12} / k^{13} ;{ }^{\mathrm{D}}(V / K)=(V / K)^{\mathrm{H}} /(V / K)^{\mathrm{D}} ;{ }^{13} K_{\mathrm{eq}}=K_{\mathrm{eq}}{ }^{12} / K_{\mathrm{eq}}{ }^{13}$. 
An intrinsic isotope effect is the isotope effect on the rate constant for a single step in a multistep reaction sequence. Observed isotope effects are often smaller than intrinsic isotope effects because other, non-isotopesensitive steps are partially rate-determining. An isotope effect on an enzymecatalyzed reaction is a function of the intrinsic isotope effect(s) for the isotope-sensitive step(s) in the overall process and of the rate of that step(s) relative to rates of other steps. Thus, intrinsic isotope effects reflect differences between ground-state and transition-state structure; observed isotope effects reflect intrinsic isotope effects and relative rates of various steps within the mechanism.

Rates of enzyme-catalyzed reactions are sometimes limited by rates of chemical transformations within the enzyme-substrate complex and sometimes by rates of dissociation of substrates or products (21). In the latter case, the substrate or product involved is said to be sticky. Operationally, this means that the rate of chemical transformation of the enzyme-substrate complex is similar to (or faster than) the rate of substrate or product dissociation.

\section{Isotope Effects and Enzyme Kinetics}

Most applications of the multiple isotope effect method involve isotope effects for elements heavier than hydrogen. Such heavy atom isotope effects are usually measured by the competitive method, in which the isotopic content (e.g. ${ }^{13} \mathrm{C} /{ }^{12} \mathrm{C}$ ) of a substrate or product is measured over the course of the reaction by means of isotope-ratio mass spectrometry (22). In careful experiments, the precision of such measurements is \pm 0.001 or better in the rate ratio. The isotope effects so obtained are those on $V / K$. Heavy-atom isotope effects on $V$ can be obtained only by separate measurement of $V$ for labeled and unlabeled substrates. This is seldom practical for heavy-atom isotope effects. Isotope effects measured by use of radioactive labels are also isotope effects on $V / K$. Isotope effects on $V / K$ reflect steps in the reaction mechanism beginning with the binding of the isotopic substrate and ending with the first irreversible step, usually the release of the first product (8).

The effect of order of addition of substrates on the observed isotope effect has been described by Klinman et al (9a), and by Cook \& Cleland (10). In a multisubstrate mechanism, if the isotopic substrate is the last substrate to bind, then the binding and dissociation of the preceding substrate(s) do not affect the isotope effect. On the other hand, when the isotopic substrate is not the last to bind, the isotope effect must be extrapolated to zero concentrations of the other substrates in order to obtain the full isotope effect.

The term rate-determining step is widely used in kinetics and has often found use in enzyme kinetics as well. Unfortunately, the term has no generally agreed-upon meaning, being used for the individual convenience of the individual investigator. The problem is exacerbated by the fact that the rate- 
determining step may change with reaction conditions, and the step that limits the overall rate may be after the first irreversible step and thus may not be reflected in the isotope effect. Several writers have tried to clarify this issue $(8,21,23)$. The tern rate-determining step should be reserved for the step whose transition state has the highest free energy; where several transition states have similar free energies, the steps can be referred to as partially rate-determining.

\section{Quantitative Relationships}

The relationship between a number of specific mechanisms and the corresponding isotope effects has been described by Cook \& Cleland (10). For a multistep mechanism of the type:

$$
\mathrm{E}+\mathrm{S} \rightleftharpoons \ldots \rightleftharpoons \mathrm{ES}_{\mathrm{n}} \underset{k_{-\mathrm{n}}}{\stackrel{k_{\mathrm{n}}}{\rightleftharpoons}} \mathrm{EP}_{1} \rightleftharpoons \ldots \rightleftharpoons \mathrm{E}+\mathrm{P}
$$

in which the only isotope-sensitive step is the step with rate constant $k_{\mathrm{n}}$, the observed isotope effect is given by:

$$
{ }^{13}(V / K)=\left[{ }^{13} k_{\mathrm{n}}+c_{\mathrm{f}}+c_{\mathrm{r}}{ }^{13} K_{\mathrm{eq}}\right] /\left[1+c_{\mathrm{f}}+c_{\mathrm{r}}\right]
$$

where ${ }^{13} k_{\mathrm{n}}$ is the isotope effect on $k_{\mathrm{n}}$, and ${ }^{13} K_{\text {eq }}$ is the isotope effect on the equilibrium constant for the overall reaction. The terms $c_{\mathrm{f}}$ and $c_{\mathrm{r}}$ contain only terms for carbon-12. The term $c_{\mathrm{f}}$ is called the "forward commitment" and reflects the rate of the isotope-sensitive step relative to the rates of preceding steps in the sequence. More specifically:

$$
c_{\mathrm{f}}=k_{\mathrm{n}} / k_{\text {substrate }}
$$

where $k_{\text {substrate }}$ is the "net rate constant" (see Cleland, 24) for the release of substrate from intermediate $\mathrm{ES}_{\mathrm{n}}$. Factor $c_{\mathrm{r}}$, the "reverse commitment," reflects the rate of reverse reaction through the isotope-sensitive step relative to release of product from complex $\mathrm{EP}_{1}$ and is defined by:

$$
c_{\mathrm{r}}=k_{-\mathrm{n}} / k_{\text {product }}
$$

where $k_{\text {product }}$ is the "net rate constant" for the release of the first product from intermediate $\mathrm{EP}_{1}$.

When the isotope-sensitive step is irreversible (e.g. in many decarboxylations), $c_{\mathrm{r}}$ goes to zero, and the equation can be simplified. 


\section{The Multiple Isotope Effect Method}

The multiple isotope effect method is applied by measuring a ${ }^{13} \mathrm{C}$ or other isotope effect, then deuterating the substrate and measuring the ${ }^{13} \mathrm{C}$ isotope effect again under the same conditions. Provided that the ${ }^{13} \mathrm{C}$ and deuterium isotope effects are significantly different from unity, deuteration may change the ${ }^{13} \mathrm{C}$ isotope effect. Deuteration is assumed to affect commitments, but not intrinsic isotope effects (a consequence of the rule of the geometric mean; see below).

It should be noted that in the analysis that follows we assume that the deuterium isotope effect is normal (i.e. $>1$ ). This will ordinarily be true for primary isotope effects, but it is not necessarily true for secondary isotope effects or for solvent isotope effects. If the deuterium isotope effect in question is inverse, then the signs of the inequalities that follow are reversed.

Deuteration of the substrate can have several effects on the ${ }^{13} \mathrm{C}$ isotope effect. The first possibility is:

$$
{ }^{13}(V / K)_{\mathrm{H}}={ }^{13}(V / K)_{\mathrm{D}}
$$

(The following subscript indicates that the substrate contains $\mathrm{H}$ or $\mathrm{D}$ at the second isotopic site.) This requires that $c_{\mathrm{f}}=c_{\mathrm{r}}=0$. In this case, $k_{\mathrm{n}}$ is the step sensitive to both ${ }^{13} \mathrm{C}$ and deuterium, and all other steps are fast compared to this step. Further, the observed carbon and deuterium isotope effects are equal to the intrinsic isotope effects. ${ }^{1}$

The second possibility is:

$$
{ }^{13}(V / K)_{H}<{ }^{13}(V / K)_{D}
$$

Thus, either $c_{\mathrm{f}}$ or $c_{\mathrm{r}}$ (or both) is decreased by deuteration. This requires that $k_{\mathrm{n}}$ (and $k_{-n}$ if the reaction is reversible) decreases on deuteration. Thus, the deuterium-sensitive step is the same as the ${ }^{13} \mathrm{C}$-sensitive step. This case differs from the first case in that other non-isotope-sensitive steps are also slow in this case.

The third and fourth possibilities begin with:

$$
{ }^{13}(V / K)_{\mathrm{H}}>{ }^{13}(V / K)_{\mathrm{D}}
$$

and deuteration has increased $c_{\mathrm{f}}$ and/or $c_{\mathrm{r}}$.

Because we are assuming that deuteration affects only a single step in the mechanism, the increase must be either in $c_{\mathrm{f}}$ (in which case, the deuteriumsensitive step precedes the ${ }^{13} \mathrm{C}$-sensitive step) or in $c_{\mathrm{r}}$ (in which case, the

\footnotetext{
'Equality of the two isotope effects can also arise from a cancellation of effects in a more complex mechanism. See the case of malate synthase below.
} 
deuterium-sensitive step follows the ${ }^{13} \mathrm{C}$-sensitive step) but not both. In the former case:

$$
\left[{ }^{13}(V / K)_{\mathrm{H}}-1\right] /\left[{ }^{13}(V / K)_{\mathrm{D}}-1\right]={ }^{\mathrm{D}}(V / K) /{ }^{\mathrm{D}} K_{\mathrm{eq}}
$$

Altematively, if the ${ }^{13} \mathrm{C}$-sensitive step is first:

$$
\left[{ }^{13}(V / K)_{\mathrm{H}}-{ }^{13} K_{\mathrm{eq}}\right] /\left[{ }^{13}(V / K)_{\mathrm{D}}-{ }^{13} K_{\mathrm{eq}}\right]={ }^{\mathrm{D}}(V / K)
$$

If the equilibrium isotope effects are known [see Cleland (25) for a compilation of equilibrium isotope effects] and are not all equal to unity, Equations 5, 6,8 , and 9 can be used to determine the order of isotope-sensitive steps.

\section{Numerical Solutions}

The second purpose of the multiple isotope effect method is to determine values for intrinsic isotope effects and relative rate constants for various steps in the mechanism, which are combined to give rise to the two commitments. Analysis of relative rates is more difficult than distinction among mechanism types. It is most successful in cases where the isotope-sensitive step is irreversible $\left(k_{-\mathrm{n}}=0\right.$, and thus $\left.c_{\mathrm{r}}=0\right)$, but even in that case, it is not invariably successful.

We expect to have experimental values for ${ }^{\mathrm{D}}(V / K),{ }^{13}(V / K)_{\mathrm{H}}$, and ${ }^{13}(V / K)_{\mathrm{D}}$. The problem comes in using these three values (and whatever other information is available) to extract intrinsic isotope effects and commitments. In case 1 above, all commitments are zero, and the intrinsic isotope effects are equal to the observed isotope effects. In case 2 (isotope-sensitive steps the same), the value of $c_{\mathrm{f}}$ for ${ }^{13}(V / K)$ is the same as the value of $c_{\mathrm{f}}$ for ${ }^{\mathrm{D}}(V / K)$, and the same is true for $c_{\mathrm{r}}$. If $c_{\mathrm{r}}$ is zero, the carbon isotope effect with deuterated substrate is given by:

$$
{ }^{13}(V / K)_{\mathrm{D}}=\left[^{13} k_{\mathrm{n}}+c_{\mathrm{f}}{ }^{\mathrm{D}} k_{\mathrm{n}}\right] /\left[1+c_{\mathrm{f}}{ }^{\mathrm{D}} k_{\mathrm{n}}\right]
$$

Thus, there are three measured isotope effects and three unknowns $\left({ }^{13} k_{n},{ }^{D} k_{n}\right.$, and $c_{\mathrm{f}}$, and the system can be solved in closed forn.

In the third and fourth cases the situation is more complex. Even if $c_{\mathrm{r}}$ is zero, the problem is that $c_{\mathrm{f}}$ for ${ }^{\mathrm{D}}(V / K)$ is not the same as $c_{\mathrm{f}}$ for ${ }^{13}(V / K)$. Additional information or additional assumptions are needed if the system is to be solved for a unique set of intrinsic isotope effects and commitments.

\section{The Rule of the Geometric Mean}

It is implicit in the equations derived above that isotope effects are independent; that is, substitution of deuterium for hydrogen in a molecule does 
not change the intrinsic carbon isotope effect, and vice versa. This is a manifestation of the rule of the geometric mean, which was originally derived experimentally and was then justified theoretically by Bigeleisen (26).

The validity of the rule of the geometric mean has been the subject of a variety of theoretical treatments (26-29). Although the rule generally works quite well, deviations are expected when vibrations involving the first isotopic site are coupled to vibrations involving the second isotopic site (27), particularly when two hydrogen isotope effects are used. Large deviations are expected when tunneling is significant $(28,29)$.

Experimentally, deviations from the rule of the geometric mean have been shown in elimination reactions involving substrates such as $\mathrm{R}-\mathrm{CL}_{2}-\mathrm{CH}_{2}-\mathrm{X}$ $(\mathrm{L}=\mathrm{H}, \mathrm{D}, \mathrm{T})(30)$ and in proton rearrangements of porphyrins (31). Deviations also occur when both isotopic substitutions are hydrogen in the enzymatic dehydrogenation of formic acid and in glucose-6-phosphate dehydrogenase (see below).

\section{ISOTOPE EFFECTS IN IRREVERSIBLE REACTIONS}

Irreversible reactions are the simplest to treat because $c_{\mathrm{r}}$ drops out of the isotope effect equation. In the following section we will consider a number of cases of this type.

\section{Single-Step Reactions}

The simplest enzyme mechanism is one involving the binding of a single substrate (or, if several substrates are involved, binding of the isotopic substrate as the last substrate), followed by a single, irreversible chemical step, followed by product dissociation. Such a reaction can be represented by:

$$
\mathrm{E}+\mathrm{S} \underset{k_{2}}{\stackrel{k_{1}}{\rightleftharpoons}} \mathrm{ES} \stackrel{k_{3}}{\longrightarrow} \mathrm{EP} \rightleftharpoons \mathrm{E}+\mathrm{P}
$$

where $k_{1}$ and $k_{2}$ are the rate constants for substrate binding and dissociation, and $k_{3}$ is that for the (irreversible) isotope-sensitive step. The isotope effect is given by:

$$
{ }^{13}(V / K)=\left[{ }^{13} k_{3}+k_{3} / k_{2}\right] /\left[1+k_{3} / k_{2}\right]
$$

The forward commitment in this case is simply $k_{3} / k_{2}$. The rate constant for substrate binding $\left(k_{1}\right)$ does not enter this equation.

If substrate dissociation from the ES complex is rapid compared to the chemical step (i.e. $k_{2}>>k_{3}$ ) then: 


$$
\begin{array}{ll}
{ }^{13}(V / K)={ }^{13} k_{3} & 13 . \\
{ }^{D}(V / K)={ }^{D} k_{3} & 14 . \\
{ }^{13}(V / K)_{H}={ }^{13}(V / K)_{D} & 15 .
\end{array}
$$

If rapid steps intervene prior to $k_{3}$, Equations 13-15 will still be true. Equation 15, in particular, is diagnostic for such cases.

FORMATE DEHYDROGENASE A clear case of this type is formate dehydrogenase, which catalyzes the reaction:

$$
\mathrm{HCO}_{2}{ }^{-}+\mathrm{NAD}^{+} \rightarrow \mathrm{CO}_{2}+\mathrm{NADH}
$$

Kinetic studies reveal that substrate binding is ordered, with $\mathrm{NAD}^{+}$binding first (32). Substrate binding and dissociation are rapid compared to catalysis.

Carbon isotope effects were measured by isotope-ratio analysis of the $\mathrm{CO}_{2}$ formed in the reaction. Hydrogen isotope effects were measured by compari-

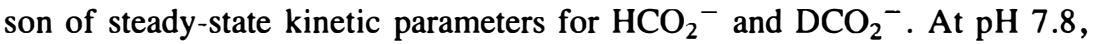
${ }^{13}(V / K)_{\mathrm{H}}=1.042 \pm 0.001,{ }^{13}(V / K)_{\mathrm{D}}=1.043 \pm 0.003(32)$, and ${ }^{\mathrm{D}}(V / K)=$ 2.2 (33). These results are consistent with the predictions of Equations 13-15 and indicate that the hydride transfer step is the only kinetically significant step (note that this eliminates mechanisms in which electron transfer is temporally separated from hydrogen-atom transfer). The isotope effects observed are the full intrinsic isotope effects. The intrinsic hydrogen isotope effect is surprisingly small and may indicate that the transition state is quite asymmetric.

Similar experiments were conducted with other nucleotides. In every case, carbon isotope effects are the same for deuterated and undeuterated formate (33).

A second application of the multiple isotope effect technique is possible in this case. Carbon- 4 of the pyridine ring of $\mathrm{NAD}^{+}$undergoes a hybridization change as a result of the hydride ransfer, and ${ }^{\mathrm{D}}(V / K)=1.23$ at this position. This value indicates that motion of this hydrogen is taking place during the hydride transfer. For $\mathrm{DCO}_{2}{ }^{-}$, the value is 1.07. According to Equation 15, we expected a value of 1.23 . The discrepancy represents a breakdown of the rule of the geometric mean and occurs because of the tunneling associated with simultaneous, coupled motion of the two hydrogens (33). Thus, caution is required when the multiple isotope effect method is applied to cases involving two hydrogens.

AMP NUCLEOSIDASE The hydrolysis of the glycosidic bond in AMP is catalyzed by the enzyme AMP nucleosidase as well as by aqueous acid:

$$
\text { adenosine-5' - } \mathrm{P}+\mathrm{H}_{2} \mathrm{O} \rightarrow \text { adenine }+ \text { ribose-5-P }
$$


Isotope effects were measured for nitrogen, carbon, and secondary hydrogen in the glycosidic bond (34). In this case, isotope effects were measured by scintillation counting of ${ }^{3} \mathrm{H} /{ }^{14} \mathrm{C}$ ratios. The precision that can be obtained in such studies is lower than that obtained by isotope-ratio measurements. An estimate of the precision of the data can be obtained by looking at the interaction of ${ }^{15} \mathrm{~N}$ and ${ }^{14} \mathrm{C}$ isotope effects. Because of the small sizes of heavy-atom isotope effects, the two isotope effects should be independent. For the acid-catalyzed reaction, ${ }^{15} k=1.030 \pm 0.002,{ }^{14} k=1.044 \pm 0.003$ for the nitrogen-14-containing substrate, and $1.033 \pm 0.005$ for the nitrogen15-containing substrate. These last two numbers should be equal. Thus, it is likely that the uncertainties in the measurements are somewhat larger than the stated experimental errors.

The principal question in this study is whether the measured isotope effects in the enzymatic reaction are totally intrinsic; that is, whether substrate and products dissociate rapidly and there is only one reaction step. ${ }^{\mathrm{D}}(V / K)$ is 1.045 \pm 0.002 and ${ }^{14}(V / K)$ is $1.035 \pm 0.002$. In a single-step reaction, we expect to see ${ }^{14}(V / K)_{\mathrm{D}}=1.035$, whereas the observed value is $1.037 \pm 0.006$.

However, the problem with this study is that the deuterium isotope effect is so small that the extent to which it might perturb the ${ }^{14} \mathrm{C}$ isotope effect is within experimental error for all possible mechanisms. If the reaction were stepwise, the ${ }^{14} \mathrm{C}$ isotope effect would be expected to decrease from 1.035 to about 1.034 , and this change would not be seen, given the precision of the measurements. This illustrates a limitation of the multiple isotope effect method: If the change in commitment caused by the perturbing isotope is too small, unattainably high precision is required to distinguish among competing mechanisms.

PREPHENATE DEHYDROGENASE Deuterium substitution does not change a ${ }^{13} \mathrm{C}$ isotope effect if the deuterium-sensitive and ${ }^{13} \mathrm{C}$-sensitive steps are the same and all preceding steps are fast (cf Equation 13). If the reaction takes place in a single chemical step, but substrate dissociation is not fast (cf Equations 6,11 , and $12 ; k_{3} / k_{2}$ decreases on deuteration), then deuteration of the substrate will increase the ${ }^{13} \mathrm{C}$ isotope effect.

An example of this type is prephenate dehydrogenase, which catalyzes the reaction:
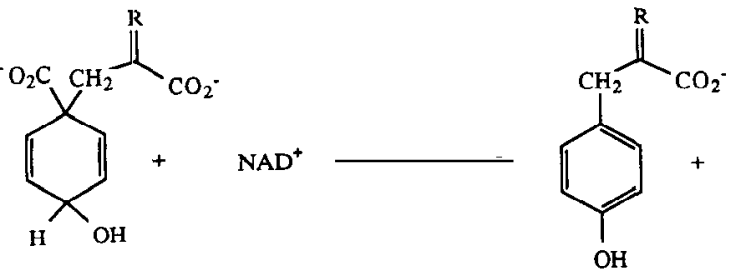

$\mathrm{CO}_{2}+\mathrm{NADH} 18$ 
The alternate substrate deoxoprephenate $\left(\mathrm{R}=\mathrm{H}_{2}\right)$ reacts at $78 \%(V)$ or $18 \%$ $(V / K)$ of the rate of prephenate $(R=0)$. Overall, the reaction involves hydride transfer, loss of $\mathrm{CO}_{2}$, and aromatization of the ring. Three mechanisms can be envisioned, depending on whether hydride transfer precedes, follows, or is simultaneous with decarboxylation. These three can be clearly distinguished by the multiple isotope effect technique.

For synthetic reasons, multiple isotope effect studies were carried out with deoxoprephenate (35). For deuteration at the position undergoing hydride transfer, ${ }^{\mathrm{D}}(V / K)=2.3,{ }^{13}(V / K)_{\mathrm{H}}=1.0033$, and ${ }^{13}(V / K)_{\mathrm{D}}=1.0103$. This result is uniquely consistent with a mechanism in which the ${ }^{13} \mathrm{C}$ - and $\mathrm{D}$ sensitive steps are the same and some other slow step precedes the chemical step (cf Equation 6). That is, decarboxylation and hydride transfer must be concerted. Because of the large thermodynamic driving force accompanying decarboxylation and aromatization, it is safe to assume that $c_{\mathrm{r}}=0$. However, the fact that deuteration increases the carbon isotope effect indicates that $c_{\mathrm{f}}$ is not zero.

With no further assumptions, it is possible to calculate ${ }^{D} k_{3}=7.3,{ }^{13} k_{3}=$ 1.015 , and $k_{3} / k_{2}=3.7$. The intrinsic carbon isotope effect is surprisingly small compared to other intrinsic isotope effects on decarboxylations, perhaps because the transition state is early in this case. It is also interesting that the reaction is concerted, since all other oxidative decarboxylations studied to date (e.g. malic enzyme, isocitrate dehydrogenase, 6-phosphogluconate dehydrogenase) are stepwise, with hydride transfer preceding decarboxylation (see below).

The isotope effects tell us that some step preceding the chemical step is partially limiting, but they do not tell us whether this step is substrate binding and dissociation or a conformation change that prepares the enzyme for catalysis. Hermes et al (35) argue for the latter, since kinetic evidence suggests that the substrates are not sticky.

\section{Stepwise Mechanisms With Both Isotope-Sensitive Steps the Same}

Most enzymatic reactions require more than a single chemical step. Many cases can be summarized as:

$$
\mathrm{E}+\mathrm{S} \underset{k_{2}}{\stackrel{k_{1}}{\rightleftharpoons}} \mathrm{ES}_{1} \underset{k_{4}}{\stackrel{k_{3}}{\rightleftharpoons}} \mathrm{ES}_{2} \stackrel{k_{5}}{\longrightarrow} \mathrm{EP} \rightleftharpoons \mathrm{E}+\mathrm{P}
$$

Where $k_{1}$ and $k_{2}$ are substrate binding and dissociation, $k_{3}$ is the first chemical step (or perhaps in the more general case, an isomerization of the ES complex), $k_{4}$ is the reverse of this step, and $k_{5}$ is the ${ }^{13} \mathrm{C}$-sensitive step. For 
the present we assume that $k_{5}$ is irreversible. If no step except $k_{5}$ is sensitive to ${ }^{13} \mathrm{C}$ substitution:

$$
{ }^{13}(V / K)=\left[{ }^{13} k_{5}+k_{5} / k_{4}\left(1+k_{3} / k_{2}\right)\right] /\left[1+k_{5} / k_{4}\left(1+k_{3} / k_{2}\right)\right]
$$

If substrate binding and dissociation are fast, this reduces to:

$$
{ }^{13}(V / K)=\left[{ }^{13} k_{5}+k_{5} / k_{4}\right] /\left[1+k_{5} / k_{4}\right]
$$

which is essentially the same as Equation 12 .

If deuteration affects $k_{5}$, then the situation is like prephenate dehydrogenase--deuteration will increase the observed ${ }^{13} \mathrm{C}$ isotope effect. However, if deuteration affects $k_{3}$ and $k_{4}$, then the ${ }^{13} \mathrm{C}$ isotope effect will decrease on deuteration and the isotope effects will obey Equation 8.

HISTIDINE DECARBOXYLASE This enzyme catalyzes the reaction:

$$
\text { histidine } \rightarrow \mathrm{CO}_{2}+\text { histamine }
$$

For the pyruvate-dependent enzyme from Lactobacillus $30 \mathrm{a},{ }^{13}(\mathrm{~V} / \mathrm{K})_{\mathrm{H}}=$ $1.0334 \pm 0.0005$. Deuteration of the substrate at the $\alpha$-position gives a small but poorly known deuterium isotope effect of perhaps 1.2 because of the change in hybridization of the $\alpha$-carbon during the decarboxylation step. For the deuterated substrate, ${ }^{13}(V / K)_{D}=1.0346 \pm 0.0002(36)$. The increase in carbon isotope effect on deuteration requires that the deuterium- and ${ }^{13} \mathrm{C}$ sensitive steps are the same.

Because ${ }^{\mathrm{D}}(V / K)$ is not accurately known, the intrinsic isotope effects cannot be approached in the same way they were above. Instead, the analysis started with a range of estimated values for the intrinsic ${ }^{13} \mathrm{C}$ isotope effect (this procedure: is probably satisfactory because of the number of analogous cases that have been studied) and used these to calculate the intrinsic deuterium isotope effect and the ratio $k_{5} / k_{4}$ (in this case, $k_{3} / k_{2}<<1$ ).

The use of a secondary isotope effect to change a ${ }^{13} \mathrm{C}$ isotope effect obeys the same equations as used above for a primary isotope effect, but the application is difficult because the secondary isotope effect is so small.

The histidine decarboxylase from Morganella morganii requires pyridoxal 5'-phosphate, rather than pyruvate, for activity. A similar analysis of this enzyme revealed a very similar set of kinetic parameters (37).

\section{Stepwise Mechanisms with Deuterium-Sensitive Step First}

If the mechanism can be expressed by Equation 19 with $k_{5}$ the ${ }^{13} \mathrm{C}$-sensitive step and $k_{3}$ and $k_{4}$ the deuterium-sensitive steps, then according to Equation 8 
the ${ }^{13} \mathrm{C}$ isotope effect decreases when the substrate is deuterated. The ${ }^{13} \mathrm{C}$ isotope effect with deuterated substrate is given by:

$$
{ }^{13}(V / K)_{\mathrm{D}}=\frac{{ }^{13} k_{5}+{ }^{\mathrm{D}} k_{4}\left(k_{5} / k_{4}\right)\left[1+\left(k_{3} / k_{2}\right) /\left({ }^{\mathrm{D}} K_{\mathrm{eq}}{ }^{\mathrm{D}} k_{4}\right)\right]}{1+{ }^{\mathrm{D}} k_{4}\left(k_{5} / k_{4}\right)\left[1+\left(k_{3} / k_{2}\right) /\left({ }^{\mathrm{D}} K_{\mathrm{eq}}{ }^{\mathrm{D}} k_{4}\right)\right]}
$$

In this case, the kinetic isotope effect on $k_{4}$ and the equilibrium deuterium isotope effect are needed. A number of deuterium isotope effects have been summarized by Cleland (25). If substrate binding and dissociation are fast, then Equation 23 simplifies to:

$$
{ }^{13}(V / K)_{\mathrm{D}}=\left[{ }^{13} k_{5}+{ }^{\mathrm{D}} k_{4}\left(k_{5} / k_{4}\right)\right] /\left[1+{ }^{\mathrm{D}} k_{4}\left(k_{5} / k_{4}\right)\right]
$$

In this case, measurement of ${ }^{13}(V / K)_{\mathrm{H}},{ }^{13}(V / K)_{\mathrm{D}}$, and ${ }^{\mathrm{D}}(V / K)$ permits calculation of ${ }^{13} k_{5},{ }^{\mathrm{D}} k_{4}$, and $k_{5} / k_{4}$. However, when substrate dissociation is slow, additional measurements or assumptions are necessary to obtain a complete solution in closed form.

MALIC ENZYME Malic enzyme catalyzes the divalent metal-dependent oxidative decarboxylation of malic acid:

$$
\text { malate }+\mathrm{NADP}^{+} \rightleftharpoons \mathrm{CO}_{2}+\text { pyruvate }+\mathrm{NADPH}
$$

The reaction is reversible, but if the pyruvate produced is removed as fast as it is formed, the reaction can be treated as being irreversible.

The reaction is believed to proceed in two steps: Oxidation of malate to form enzyme-bound oxalacetate, followed by decarboxylation. According to Equation 8, deuteration of carbon- 2 of malate should decrease the observed isotope effect. On the other hand, if the reaction is concerted, the carbon isotope effect will stay the same or increase on deuteration.

The measured isotope effects are ${ }^{13}(V / K)_{\mathrm{H}}=1.031,{ }^{13}(V / K)_{\mathrm{D}}=1.025$, and $\mathrm{D}_{(V / K)}=1.47(15)$. This is consistent with the expectation that hydride transfer precedes decarboxylation and that enzyme-bound oxalacetate is an intermediate. The fit of the isotope effects to Equation 8 is excellent.

A complete solution for the intrinsic isotope effects and rate ratios requires additional information. Grissom \& Cleland (38) generated the enzymeNADPH-oxalacetate complex and measured its partitioning between product formation and release of starting materials. This procedure was repeated with deuterated nucleotide. A complete set of kinetic parameters can then be derived: ${ }^{13} k_{5}=1.044 ;{ }^{\mathrm{D}}(V / K)=5.7 ; k_{5} / k_{4}=10 ; k_{3} / k_{2}=3.3$. The latter ratio 
may also include a contribution from an enzyme conformation change in $\boldsymbol{k}_{\mathbf{1}}$ and $k_{2}$. Interestingly, the intrinsic carbon isotope effect is only slightly different from the value of 1.049 for the $\mathrm{Mg}^{2+}$-catalyzed decarboxylation of oxalacetate (39).

The enzyme from the plant Crassula argenta gives similar isotope effects (40).

ISOCITRATE DEHYDROGENASE The oxidative decarboxylation of isocitric acid is chemically similar to the oxidative decarboxylation of malic acid in that an enzyme-bound beta-ketoacid is believed to be an intermediate. The overall reaction is:

$$
\text { Isocitrate }+\mathrm{NADP} \rightleftharpoons \mathrm{CO}_{2}+\text { 2-ketoglutarate }+\mathrm{NADPH}
$$

The catalytic mechanism is random sequential, and catalysis seems to be more rapid than product release $(41,42)$. As expected for such a mechanism, isotope effects under optimum conditions are small: ${ }^{13}(V / K)=0.9989(43)$ and ${ }^{\mathrm{D}}\left(V / K^{\prime}\right)=1.00(10)$. Isotope effects of this magnitude cannot be used safely in the multiple isotope method. However, as the $\mathrm{pH}$ is lowered, the carbon isotope effect increases. The largest value obtained was ${ }^{13}(V / K)_{\mathrm{H}}=$ 1.028 at pH 4.1, and ${ }^{13}(V / K)_{\mathrm{D}}=1.005(44)$. Because of instability of enzyme and nucleotide, these values could be obtained only with difficulty, and values at still lower $\mathrm{pH}$ could not be measured. The carbon isotope effects were fitted to limiting isotope effects at low $\mathrm{pH}$ of ${ }^{13}(V / K)_{H}=1.04$ and ${ }^{13}(V / K)_{\mathrm{D}}=1.016$. The deuterium isotope effect increases only slightly with decreasing $\mathrm{pH}$. Qualitatively, it appears that hydride transfer and decarboxylation are separate steps, but these data do not fit Equation 8, and the reason is not known.

6-PHOSPHOGLUCONATE DEHYDROGENASE This enzyme catalyzes a reaction that is formally similar to the previous two:

$$
\text { 6-phosphogluconate }+\mathrm{NADP}^{+} \rightleftharpoons \mathrm{CO}_{2}+\text { ribulose 5-P + NADPH } 27 .
$$

The mechanism may proceed in a stepwise fashion, with hydride transfer first, followed by decarboxylation. Consistent with this expectation, ${ }^{\mathrm{D}}(V / K)$ $=1.61,{ }^{13}(V / K)_{\mathrm{H}}=1.0096$, and ${ }^{13}(V / K)_{\mathrm{D}}=1.0081(45)$. Sufficient data are not available to permit calculation of unique intrinsic isotope effects.

DIHYDROOROTATE OXIDASE Two deuterium isotope effects can also be used in multiple isotope effect studies. The enzyme catalyzes the reaction: 
<smiles>CC1C(=O)NC(=O)NC1C(=O)[O-]</smiles><smiles>O=C([O-])c1cc(=O)[nH]c(=O)[nH]1</smiles>

28.

The elimination of the two hydrogens is from the anti positions (46). Pascal \& Walsh (46) synthesized various deuterated forms of the substrate and measured the rates. For deuteration at $\mathrm{H}_{5},{ }^{\mathrm{D}}(V / K)=2.93$; at $\mathrm{H}_{4},{ }^{\mathrm{D}}(V / K)=2.99$. When substrate deuterated at $\mathrm{H}_{5}$ is used, the isotope effect at $\mathrm{H}_{4}$ is reduced to 2.09. Qualitatively, this is consistent with a stepwise mechanism for hydrogen removal (Equations 8 and 9) but not with a concerted mechanism (Equations 5 and 6). Unfortunately, it is not possible to use the isotope effects to determine which hydrogen is removed first. Isotope exchange studies suggest that the hydrogen at C-5 is removed first (46).

MALATE SYNTHASE This enzyme catalyzes the reaction:

$$
\text { acetyl } \mathrm{CoA}+\text { glyoxylate } \rightleftharpoons \text { malate }+\mathrm{CoA}
$$

Qualitatively, the mechanism involves hydrogen abstraction from acetyl CoA followed by (or concomitant with) nucleophilic attack on carbon- 2 of glyoxylate. The question is whether the mechanism is synchronous or stepwise. The enzyme does not catalyze hydrogen exchange into the methyl group of acetyl CoA (47), but that is an unreliable indicator of mechanism, since the abstracted proton might be sequestered in the active site.

Trideutero acetyl CoA gives ${ }^{\mathrm{D}}(V / K)=1.3$ (47). This value represents the product of the primary isotope effect for the hydrogen being transferred and the secondary isotope effects for the two hydrogens remaining behind. In spite of this complication, this is the proper isotope effect to use in the multiple isotope effect study. The inramolecular kinetic isotope effect using $\mathrm{CH}_{2} \mathrm{D}$ acetyl CoA is $3.8(48)$, and this value gives a lower limit on the intrinsic hydrogen isotope effect for the hydrogen being transferred.

Carbon isotope effects for carbon-2 of glyoxylate are ${ }^{13}(V / K)_{\mathrm{H}}=1.0037 \pm$ 0.0004 and ${ }^{13}(V / K)_{D}=1.0037 \pm 0.0007$ with trideuterated acetyl CoA. These values are so small that they are unlikely to be intrinsic isotope effects.

The equality of carbon isotope effects is at first glance most consistent with a single-step mechanism in which hydrogen transfer and carbon-carbon bond formation are synchronous and all other steps are fast. However, if that mechanism were correct, then the observed hydrogen isotope effect should be equal to the intrinsic isotope effect, and that is not the case. 
Clark et. al (47) argue that if the mechanism were concerted, the carbon isotope effect should increase to about 1.011 on deuteration of acetyl CoA; instead, the mechanism must be stepwise. The lack of a deuterium effect on the ${ }^{13} \mathrm{C}$ isotope effect may be due to the occurrence of an early ratedetermining step that is not isotope sensitive.

Interpretation of the carbon isotope effects is made more difficult by the fact that glyoxylate is at least $\mathbf{9 9 \%}$ hydrated in aqueous solution, and there may be an equilibrium carbon isotope effect on that hydration. The unhydrated aldehyde is presumably the substrate for the condensation reaction. If the carbon isotope effect on the hydration were approximately 1.003 , then the corrected carbon isotope effects would be approximately 1.000 , and the multiple isotope effect method could not be applied. However, even in this case, the conclusion that the reaction is stepwise would still stand.

RIBULOSE BISPHOSPHATE CARBOXYLASE The first step in the $\mathrm{C}_{3}$ pathway of photosynthesis is catalyzed by the enzyme ribulose bisphosphate carboxylase:

Ribulose-1,5-bisphosphate $+\mathrm{CO}_{2} \rightarrow 2$ 3-phosphoglyceric acid 30.

A variety of mechanistic information suggests that the reaction is stepwise (49): A hydrogen is first abstracted from carbon- 3 of the substrate, and at this position ${ }^{\mathrm{D}}(V / K)=2.1$ at $\mathrm{pH} 8(50)$. Following a proton transfer within the intermediate, reaction with $\mathrm{CO}_{2}$ occurs. Carbon isotope effects are ${ }^{13}(V / K)_{\mathrm{H}}=1.029 \pm 0.001$ and ${ }^{13}(V / K)_{\mathrm{D}}=1.021 \pm 0.003(51)$. These values are qualitatively consistent with the stepwise mechanism, but they do not fit Equation 8, probably because of the occurrence of a second deuteriumsensitive step following carboxylation.

\section{Stepwise Mechanisms with the ${ }^{13}$ C-Sensitive Step First}

Such cases are expected to fit Equation 9. Although specific examples have not been fitted to this mechanism, note that reactions such as malic enzyme, which fit Equation 8 in the forward direction, will fit Equation 9 in the reverse direction.

\section{Stepwise Mechanisms with Two Isotope-Sensitive Steps}

If two steps are deuterium sensitive or if two steps are heavy-atom sensitive, then Equations 5-9 can no longer be used. If the relative sizes of the isotope effects on the two steps can be predicted (if they are expected to be equal, or if one should clearly be larger than the other), then a set of predictions analogous to those used above can be made. This situation is likely to be 
common for nitrogen isotope effects and when solvent isotope effects are used to perturb the heavy-atom isotope effect.

PHENYLALANINE AMMONIA-LYASE This enzyme catalyzes the reaction:

$$
\text { Phenylalanine } \rightleftharpoons \mathrm{NH}_{4}{ }^{+}+\text {cinnamate }
$$

The enzyme contains a dehydroalanine cofactor (52) that is presumed to activate the nitrogen atom of the substrate by formation of a covalent bond. Cinnamate is released from the enzyme before ammonia, possibly because hydrolysis of the covalent dehydroalanine-ammonia complex is slow (52).

The nitrogen-15 isotope effect was measured by isolation of released ammonia and conversion to molecular nitrogen for isotope-ratio analysis. Studies with phenylalanine were not very satisfactory. Isotope effects were small $\left({ }^{\mathrm{D}}(V / K)=1.15\right.$ at carbon-3), presumably because dissociation of substrates and/or products is slow. Instead, isotope effect studies were carried out with 2,5-dihydrophenylalanine, which reacts at about $7 \%$ the rate of phenylalanine; thus, we expect that in this case, substrate and product dissociation are fast compared to catalysis. For this compound, ${ }^{\mathrm{D}}(V / K)=2.0$, and this value is $\mathrm{pH}$ independent. The value of ${ }^{15}(V / K)$ increases with decreasing $\mathrm{pH}$ because of the acid-base equilibrium involving the substrate amino group $\left({ }^{15} K_{\mathrm{a}}=1.016\right)$. When the isotope effect is calculated for unprotonated substrate, ${ }^{15}(V / K)_{\mathrm{H}}=1.0047$, and ${ }^{15}(V / K)_{\mathrm{D}}=0.9921(53)$. These results indicate that the reaction is stepwise.

The quantitative analysis is more complex in this case than in previous cases for a couple of reasons. First, the reaction is reversible and it may not be safe to assume that the first $\mathrm{C}-\mathrm{N}$ bond-breaking step is irreversible. Second, at least two steps have significant nitrogen isotope effects: the step in which the covalent complex between substrate and dehydroalanine is formed, and the step in which the substrate $\mathrm{C}-\mathrm{N}$ bond is broken. Hermes et al (53) argue that the mechanism is stepwise, with $\mathrm{C}-\mathrm{H}$ bond breaking preceding $\mathrm{C}-\mathrm{N}$ bond breaking.

\section{REVERSIBLE REACTIONS}

The qualitative distinction among the three classes of mechanisms (Equations 5-9) still holds, whether or not the second isotope-sensitive step is reversible, provided the experiments are conducted in such a way that the release of the first product from the enzyme is irreversible. In fact, it is possible that some of the cases above should have been considered reversible within this context (e.g. malic enzyme and isocitrate dehydrogenase), but this change would not affect the conclusion about concerted vs stepwise mechanisms. 
Quantitative analysis of reversible reactions is difficult. For most reversible reactions, the number of experimental observables is inadequate to provide a unique set of intrinsic isotope effects and rate constant ratios. The cases below illustrate a variety of approaches to this problem.

GLUCOSE-6-PHOSPHATE DEHYDROGENASE The reaction catalyzed is:

Glucose-6-phosphate + NADP $^{+} \rightleftharpoons$ 6-phosphogluconolactone + NADPH

Although the equilibrium lies on the right, hydride transfer is reversible. The isotope effects are ${ }^{\mathrm{D}}(V / K)=2.97$ for the hydrogen being transferred, ${ }^{13}(V / K)_{\mathrm{H}}=1.0165$, and ${ }^{13}(V / K)_{\mathrm{D}}=1.0316(15)$ for carbon-1. The increase in carbon isotope effect on deuteration indicates that the reaction is concerted, with the ${ }^{13} \mathrm{C}$ - and deuterium-sensitive steps the same. The hydrogen isotope effect was also measured for the 4-position of the pyridine ring of $\mathrm{NADP}^{+}$. Narrow limits could be calculated for the various intrinsic isotope effects and rate ratios.

In a subsequent study, the same isotope effects were measured in $\mathrm{D}_{2} \mathrm{O}(54)$. In this solvent, ${ }^{13}(V / K)_{\mathrm{H}}=1.0110,{ }^{13}(V / K)_{\mathrm{D}}=1.0242$, and ${ }^{\mathrm{D}}(V / K)=1.81$. The intrinsic hydrogen isotope effect (for the hydrogen being transferred) appears to be smaller in $\mathrm{D}_{2} \mathrm{O}$ than in $\mathrm{H}_{2} \mathrm{O}$. As in the case of formate dehydrogenase (see above), coupled motion of more than a single hydrogen in the transition state can lead to a breakdown of the rule of the mean. The analysis is somewhat complicated because the effects of $\mathrm{D}_{2} \mathrm{O}$ are primarily on steps other than the chemical step (binding and dissociation or conformation change steps), but the fundamental analysis is probably correct.

TRANSCARBOXYLASE O'Keefe \& Knowles $(55,56)$ studied the biotindependent carboxylation of pyruvate by malonyl CoA:

$$
\text { pyruvate }+ \text { malonyl } \mathrm{CoA} \rightleftharpoons \text { oxalacetate }+ \text { acetyl } \mathrm{CoA}
$$

The carbon isotope effect was obtained by reduction of oxalacetate to malate, and then isotopic analysis of carbon- 4 of malate. For trideuteropyruvate, ${ }^{\mathrm{D}}(V / K)=1.39$. For the carbon being transferred, ${ }^{13}(V / K)_{\mathrm{H}}=1.0227 \pm$ 0.0008 and ${ }^{13}(V / K)_{\mathrm{D}}=1.0141 \pm 0.001$.

The isotope effects fit Equation 8. Presumably, enolization of pyruvate is the first step, and this is followed by carboxylation. However, the isotope effects do not distinguish between a reaction with direct carboxyl transfer between carboxybiotin and the enolate and a transfer involving enzyme-bound $\mathrm{CO}_{2}$. 
PROLINE RACEMASE This case is complex because the reaction is, of course, reversible. Extensive kinetic and isotope effect studies have been reported by Knowles and collaborators $(16,17,57-60)$. The reaction is:

\section{D-proline $\rightleftharpoons$ L-proline}

34.

The enzyme operates by a two-base mechanism: one base abstracts the hydrogen from one face of the substrate, and the other base donates a hydrogen to the other face. In the presence of substrate, the hydrogen bound to one enzyme site cannot exchange with the solvent and cannot be transferred to the other site. At high substrate concentrations', hydrogen transfer between these two sites in the free enzyme may become rate-determining.

The multiple isotope effect approach can be used to determine whether the two hydrogen transfers are simultaneous or stepwise. However, because one of the hydrogens involved is ultimately derived from the solvent, the experimental design is more complex. The isotope effect was measured by running the reaction in an $\mathrm{H}_{2} \mathrm{O} / \mathrm{D}_{2} \mathrm{O}$ mixture and then comparing the deuterium content of the product with that of the solvent. The same measurement was then conducted with the deuterated substrate. The reaction was made nearly irreversible by running to only about $15 \%$ completion.

With proline, the isotope effect for hydrogen incorporation from solvent was 2.78 . With deuterated proline the value was $2.70(16,17)$. The mechanism of this reaction involves two partially rate-determining steps, and both steps have significant hydrogen isotope effects at both sites. This apparently occurs because the $t$.es at both sites are thiols. Thiols show an equilibrium isotope effect of about $2 \mathrm{X}$ for the exchange reaction:

$$
\mathrm{HOD}+\mathrm{R}-\mathrm{SH} \rightleftharpoons \mathrm{HOH}+\mathrm{R}-\mathrm{SD}
$$

Thus, both steps show hydrogen isotope effects at both sites. The first step will show a primary hydrogen isotope effect at one site and an equilibrium isotope effect at the other. In the second step, these roles will be reversed. The equilibrium fractionation into the enzyme site was confirmed in separate experiments (58).

\section{APPLICATIONS USING SOLVENT ISOTOPE EFFECTS}

As we noted earlier, the multiple isotope effect method works because the second isotopic substitution represents a minimal perturbation of the chemical and kinetic properties of the system. Changing the solvent from $\mathrm{H}_{2} \mathrm{O}$ to $\mathrm{D}_{2} \mathrm{O}$ can be used in the same way, but some significant problems arise.

First, changing from $\mathrm{H}_{2} \mathrm{O}$ to $\mathrm{D}_{2} \mathrm{O}$ may change the rates of several steps in a multistep mechanism. Further, $\mathrm{D}_{2} \mathrm{O}$ can affect substrate binding, so $\mathrm{D}_{2} \mathrm{O}$ 
effects on $V / K$ cannot safely be used in the same way as substrate deuterium effects on $V / K$. One is tempted to use solvent isotope effects on $V$, which are independent of substrate binding, but $V$ may include steps subsequent to the first irreversible step, and thus may not provide a proper comparison.

Second, $\mathrm{D}_{2} \mathrm{O}$ can affect $\mathrm{pK}_{\mathrm{a}}$ values for catalytic groups on the enzyme. Procedures for including this effect are well understood (9), but measurements at a single $\mathrm{pH}(\mathrm{pD})$ must be interpreted with caution.

Third, the validity of the rule of the geomerric mean in $\mathrm{H}_{2} \mathrm{O} / \mathrm{D}_{2} \mathrm{O}$ comparisons must be considered. A consequence of the rule of the mean is that geomerry must be invariant with isotopic substitution. Protein conformation might be altered by substitution of $\mathrm{D}_{2} \mathrm{O}$ for $\mathrm{H}_{2} \mathrm{O}$, with a corresponding change in intrinsic isotope effects, but several lines of evidence suggest that this is not so. First, solvent isotope effects on enzymatic reactions are generally of the magnitude expected from consideration of organic reactions; they are not significantly larger, as would have been expected if these conformational issues were important. Second, proton-inventory studies of a variety of enzymes are consistent with the concept that solvent isotope effects on enzymatic reactions are usually derived from one or two significant hydrogens, rather than from a large number (9). Thus, we assume for the present that intrinsic isotope effects are the same in $\mathrm{H}_{2} \mathrm{O}$ as in $\mathrm{D}_{2} \mathrm{O}$ [except for cases in which the rule of the mean breaks down due to multiple coupled hydrogen motion (54)].

\section{Theory}

Solvent isotope effects on enzymatic reactions have been discussed by Venkatasubban \& Schowen (9). For multiple isotope effect studies, the best approach is to write equations analogous to Equation 2 for both solvent species and then try to make reasonable assumptions about the solvent isotope effect on each reaction step.

Several different experimental approaches have been used for studying solvent isotope effects on other isotope effects. In the case of proline racemase described above, deuteration of a primary site was used to change the primary isotope effect obtained in an $\mathrm{H}_{2} \mathrm{O} / \mathrm{D}_{2} \mathrm{O}$ mixture. In the case of alcohol dehydrogenase described below, deuteration of the substrate was used to perturb the solvent isotope effect. In other cases, heavy-atom isotope effects have been compared in $\mathrm{H}_{2} \mathrm{O}$ and in $\mathrm{D}_{2} \mathrm{O}$.

YEAST ALCOHOL DEHYDROGENASE Yeast alcohol dehydrogenase catalyzes the reduction of a number of aromatic aldehydes:

$$
\mathrm{Ar}-\mathrm{CHO}+\mathrm{NADH} \rightleftharpoons \mathrm{Ar}-\mathrm{CH}_{2} \mathrm{OH}+\mathrm{NAD}^{+}
$$

For acetaldehyde and similar substrates, catalysis is faster than substrate and 
product dissociation (61). For aromatic aldehydes, catalysis is limiting. For example, p-methoxybenzaldehyde gives a primary hydrogen isotope effect for reduction by NADD of $3.6(62)$. The corresponding primary tritium effect is 6.3 , which obeys the Swain-Schaad relationship (63) and suggests that the hydride transfer step is fully rate-limiting (13). Consistent with this is the observation that the solvent isotope effect on the reduction is $0.50 \pm 0.05$ for reduction by NADH and $0.58 \pm 0.06$ for reduction by NADD (62).

Thus, the hydride transfer step must be fully rate-limiting, and this reaction corresponds to case 1 above. Interestingly, these results also require that NADH dissociation is rapid compared to catalysis.

GLUTAMATE DECARBOXYLASE The pyridoxal phosphate-dependent glutamate decarboxylase from $E$. coli catalyzes the irreversible reaction:

$$
\text { glutamate } \rightarrow \mathrm{CO}_{2}+\mathrm{GABA}
$$

In $\mathrm{H}_{2} \mathrm{O},{ }^{13}(V / K)=1.018$, whereas in $\mathrm{D}_{2} \mathrm{O},{ }^{13}(V / K)=1.009(64)$. Solvent isotope effects are large: ${ }^{\mathrm{D}_{2}} \mathrm{O}(V / K)=2.6$, and ${ }^{\mathrm{D}_{2} \mathrm{O}} V=5.0$. A proton-inventory analysis shows that the isotope effect on $V$ reflects contributions from several hydrogens.

Substrate dissociation is rapid and decarboxylation is irreversible, so the reaction can be treated as having two kinetically significant steps (cf Equations 19 and 21). Models indicate that the intrinsic carbon isotope effect should be about $1.06(65)$. With this value in hand, it is possible without further assumptions to calculate a ratio of solvent isotope effects on $k_{5}$ (the decarboxylation step) and $k_{4}$ (the Schiff-base interchange step). Using the steady-state solvent isotope effects, we estimate that the solvent isotope effect is about 3 on decarboxylation and about 7 on Schiff-base interchange. The latter isotope effect may be due to the fact that a large number of protons change places in this step and/or to the fact that Schiff-base interchange involves a conformation change. Nitrogen isotope effects on this reaction are also consistent with this mechanism (66).

Similar studies have been carried out for histidine decarboxylase (36).

BENZOYLFORMATE DECARBOXYLASE This enzyme is similar to pyruvate decarboxylase and catalyzes the thiamine pyrophosphate-dependent reaction:

$$
\mathrm{Ar}-\mathrm{CO}-\mathrm{CO}_{2}{ }^{-} \rightarrow \mathrm{Ar}-\mathrm{CHO}+\mathrm{CO}_{2}
$$

The substrate first reacts with the enzyme-bound cofactor to form a tetrahedral adduct, which then undergoes decarboxylation.

Carbon isotope effects were measured in $\mathrm{H}_{2} \mathrm{O}$ and in $\mathrm{D}_{2} \mathrm{O}$ for five substrates with ring substituents covering a range of electron-withdrawing 
capacities. The values in $\mathrm{H}_{2} \mathrm{O}$ range from 1.002 to 1.019. Values in $\mathrm{D}_{2} \mathrm{O}$ were about half the corresponding values in $\mathrm{H}_{2} \mathrm{O}$. Solvent isotope effects on $V / K$ ranged from 1.4 to 3.1 (67).

Using an intrinsic carbon isotope effect of $1.051(68)$, we can calculate that the ratio of the solvent isotope effect on the decarboxylation step to that on the preceding steps is about 0.5 , probably because there is a relatively large solvent isotope effect on formation of the tetrahedral adduct. This is not surprising, since formation of the adduct involves at least one proton transfer from the solvent. Attempts to fit the carbon isotope effects to Equation 8 were not successful, presumably because several steps show significant solvent isotope effects (67).

ADENOSINE DEAMINASE The reaction catalyzed is:

$$
\text { adenosine } \rightarrow \mathrm{NH}_{3}+\text { inosine }
$$

The mechanism appears to involve an addition-elimination sequence at the site of substitution on the adenine ring. Chemical modification and $\mathrm{pH}$ studies have been interpreted to indicate that an enzyme sulfhydryl group is involved in the reaction (69).

For adenosine, ${ }^{15}(V / K)=1.0048$ in $\mathrm{H}_{2} \mathrm{O}$ and 1.0023 in $\mathrm{D}_{2} \mathrm{O}$. The values are $\mathrm{pH}$ independent, even though $\mathrm{V} / \mathrm{K}$ is $\mathrm{pH}$ dependent. The solvent isotope effect on $V / K$ is 0.77 .

Larger isotope effects were obtained with 7,8-dihydro-8-oxoadenosine, which reacts at $10 \%$ of the rate of adenosine. For this compound, ${ }^{15}(V / K)=$ 1.0150 in $\mathrm{H}_{2} \mathrm{O}$ and 1.0131 in $\mathrm{D}_{2} \mathrm{O}$. The solvent isotope effect is 0.45 on $V / K$. The inverse solvent isotope effect probably indicates that a sulfhydryl group acts as a proton donor at the active site of the enzyme.

The fact that the nitrogen isotope effect decreases in $\mathrm{D}_{2} \mathrm{O}$ rules out mechanisms in which there is only a single kinetically significant step. From isotope effects and other evidence, the reaction appears to have at least two kinetically significant steps: first, addition of some nucleophile to form a tetrahedral adduct, then loss of ammonia from this adduct. The fact that the nitrogen isotope effect is smaller in $\mathrm{D}_{2} \mathrm{O}$ suggests that the solvent isotope effect on the C-N bond cleavage step is smaller than that on some other step. At some point (probably prior to the first step) the nitrogen must be protonated, and this protonation can lead to a nitrogen isotope effect of about 1.016. A similar analysis has been conducted for alanine dehydrogenase (70).

CARBONIC ANHYDRASE The reversible dehydration of bicarbonate is catalyzed by carbonic anhydrase:

$$
\mathrm{HCO}_{3}^{-}+\mathrm{H}^{+} \rightleftharpoons \mathrm{CO}_{2}+\mathrm{H}_{2} \mathrm{O}
$$


The enzyme requires $\mathrm{Zn}^{2+}$ for activity. The kinetic mechanism appears to be ping-pong, with the dehydration half reaction separated from the proton ransfer between enzyme and solvent. The solvent isotope effect on $V$ is about 5 because the proton transfer is rate-determining (71).

The carbon isotope effect on the dehydration of $\mathrm{HCO}_{3}{ }^{-}$is 1.010 , and this value is not changed if $\mathrm{D}_{2} \mathrm{O}$ is substituted for $\mathrm{H}_{2} \mathrm{O}$, if $\mathrm{Co}^{2+}$ is substituted for $\mathrm{Zn}^{2+}$, or if the viscosity of the solvent is changed (72). The measured isotope effect is only slightly larger than the equilibrium isotope effect on dehydration.

The lack of a solvent isotope effect on the carbon isotope effect is consistent with the ping-pong mechanism; otherwise, the carbon isotope effect should change in $\mathrm{D}_{2} \mathrm{O}$. The isotope effect is approximately equal to that seen in the nonenzymatic loss of $\mathrm{OH}^{-}$from $\mathrm{HCO}_{3}{ }^{-}$(P. Paneth, M. H. O'Leary, unpublished), and this, together with the lack of a $\mathrm{D}_{2} \mathrm{O}$ effect on the carbon isotope effect, suggests that the dehydration step is rate-limiting in the dehydration half-reaction.

PYRUVATE CARBOXYLASE The biotin-dependent carboxylation of pyruvic acid occurs in two stages: The first is the ATP-dependent carboxylation of biotin, and the second is carboxylation of pyruvate (presumably as the enol or enolate) to form oxalacetate. The reaction is reversible. Attwood et al (73) studied the second half-reaction in the reverse direction:

$$
\text { oxalacetate } \rightarrow \mathrm{CO}_{2}+\text { pyruvate }
$$

which occurs by transfer of carbon- 4 to biotin, and then decomposition of carboxybiotin.

In $\mathrm{H}_{2} \mathrm{O},{ }^{13}(\mathrm{~V} / \mathrm{K})$ is 1.032 ; the value in $\mathrm{D}_{2} \mathrm{O}$ is 1.025 . The solvent isotope effect on $V / K$ is 2.1 . When the reaction is run in the opposite direction, trideuteropyruvate gives an isotope effect of 2.8. Dissociation of oxalacetate from the enzyme-substrate complex is fast (74). It appears that the carboxylation is stepwise, as suggested above for the case of transcarboxylase.

\section{CONCLUSIONS AND PROSPECTS}

The change in an observed isotope effect as a result of a second isotopic substitution can often be used to learn about the order of the isotope-sensitive steps. In favorable cases, intrinsic isotope effects and rate ratios can be extracted from the data. This is most practical in reactions in which one of the isotope-sensitive steps is irreversible. The ideal cases are ones in which a primary deuterium isotope effect is used to perturb a ${ }^{13} \mathrm{C}$ isotope effect.

The assumption that only one step in the reaction mechanism is affected by each isotopic substitution is an important one. When this is not so, Equations 
5-9 do not apply and it is more difficult to solve for intrinsic isotope effects and rate ratios.

\section{Precision and Uncertainty}

Heavy-atom isotope effects are always small; the uninitiated accuse practitioners of making "much ado about nothing." Although heavy-atom isotope effects can be measured with a reproducibility of \pm 0.001 or better, not all reported data are of that quality. Careful attention to experimental detail is essential. Separate experiments must be statistically independent (22). Systematic errors have always been a serious problem in heavy-atom isotope effect studies, and it is not always clear that these have been eliminated.

Problems are even more severe when the perturbing isotope has only a small isotope effect and thus can cause only a small change in the original isotope effect. Problems also arise when the original isotope effect is much smaller than the expected intrinsic value. In that case, the change in isotope effect due to the second isotopic substitution becomes quite small and the precision of measurement may not be adequate to permit a clear distinction among mechanisms. The magnitudes of isotope effects can often be increased by working on the limbs of the $\mathrm{pH}$-rate profile.

\section{Reversibility}

Perhaps the most serious increase in complexity in the multiple isotope effect method occurs when we move from reactions in which one of the isotopesensitive steps is irreversible to reactions in which all steps up to the release of the first product are reversible. This change does not affect distinctions between concerted and stepwise mechanisms based on Equations 5-9, but it does affect our ability to calculate individual isotope effects and rate ratios.

The most serious problem is more subtle than this. The mathematical treatment given above assumes that the release of the first product is irreversible and that subsequent steps need not be considered. The history of this assumption is that it is derived from steady-state kinetics, where initial rates are measured, and the concentrations of products are effectively zero in the experiments. However, the same conditions do not necessarily hold in the measurement of heavy-atom isotope effects. When isotope effects are measured by the analysis of products, the initial measurement is usually taken at $10-15 \%$ reaction, and occasionally as high as $25 \%$ reaction. Even at $10 \%$ reaction, an appreciable amount of product has accumulated, and if the equilibrium constant for the reaction is not too far from unity, a significant amount of reverse reaction may occur. This will cause the observed isotope effect to be smaller than the actual isotope effect.

Even in the case of malic enzyme, where the equilibrium favors decarboxylation, this can be a problem. In early measurements of the carbon isotope effect, values near 1.02 were obtained. When lactate dehydrogenase 
and NADH were added, so that pyruvate release became irreversible, the isotope effect increased to 1.03 (15). We are left to wonder whether decarboxylation and $\mathrm{CO}_{2}$ release might be reversible in other cases. If so, then the measured isotope effect will be smaller than the actual effect.

\section{Theoretical Limitations}

The principal theoretical limitation on the use of the multiple isotope effect method is the limitation on the applicability of the rule of the geometric mean. When this rule fails, the observed isotope effect will change upon isotopic substitution, even if the reaction in question involves only a single step.

Failure of the rule of the geometric mean may be fairly common in cases in which the effect of hydrogen/deuterium substitution on a hydrogen isotope effect is considered. If the sites are sufficiently close together that both give rise to isotope effects, then the sites are also sufficiently close together that vibrational coupling will occur, and the rule of the geometric mean will break down.

It is becoming clear that a number of enzymatic reactions that involve hydrogen transfer also involve a significant degree of hydrogen tunneling in the hydrogen transfer transition state. When tunneling occurs, then the rule of the geometric mean may break down, particularly when the second isotopic substitution also involves hydrogen. The importance of the analogous phenomenon in multiple isotope effect studies using heavy-atom isotope effects is not currently known.

\section{Literature Cited}

1. Bigeleisen, J., Wolfsberg, M. 1958. Adv. Chem. Phys. 1:15-76

2. Melander, L., Saundcrs, W. H. Jr. 1980. Reaction Rates of Isotopic Molecules. New York: Wiley-Interscience

3. Cleland, W. W., O'Leary, M. H., Northrop, D. B. 1977. Isotope Effects on Enzyme-Catalyzed Reactions. Baltimore: Univ. Park Press

4. Klinman, J. P. 1978. Adv. Enzymol. 46:415-94

5. O'Leary, M. H. 1978. Transition States of Biochemical Processes, ed. R. D. Gandour, R. L. Schowen, pp. 285-316. New York: Plenum

6. Cleland, W. W. 1982. CRC Crit. Rev. Biochem. 13:385-428

7. Cleland, W. W. 1987. Bioorg. Chem. 15:283-302

8. Northrop, D. B. 1981. Annu. Rev. Biochem. 50:103-31

9. Venkatasubban, K. S., Schowen, R. L. 1984. CRC Crit. Rev. Biochem. 17:1-44

9a. Klinman, J. P., Humphries, H., Voet, J. G. 1980. J. Biol. Chem. 255:1164851
10. Cook, P. F., Cleland, W. W. 1981. Biochemistry 20:1790-96

11. Cook, P. F., Cleland, W. W. 1981. Biochemistry 20:1797-805

12. Cook, P. F., Cleland, W. W. 1981. Biochemistry 20:1805-16

13. Northrop, D. B. 1975. Biochemistry $14: 2644-51$

14. O'Leary, M. H., Marlier, J. F. 1979. J. Am. Chem. Soc. 101:3300-6

15. Hermes, J. D., Roeske, C. A., O'Leary, M. H., Cleland, W. W. 1982. Biochemistry 21:5106-14

16. Belasco, J. G., Albery, W. J., Knowles, J. R. 1983. J. Am. Chem. Soc. 105:2475-77

17. Belasco, J. G., Albery, W. J., Knowles, J. R. 1986. Biochemistry 25:2552-58

18. Koch, H. F., Dahlberg, D. B. 1980. J. Am. Chem. Soc. 102:6102-7

19. Koch, H. F., McLennan, D. J., Koch, J. G., Tumas, W., Dobson, B., Koch, N. H. 1983. J. Am. Chem. Soc. 105:193037

20. Northrop, D. B. 1977. See Ref. 3, pp. 122-57 
21. Cleland. W. W. 1975. Acc. Chem. Res. 8:145-51

22. O'Leary, M. H. 1980. Methods Enzymol. 154:83-104

23. Ray, W. J. Jr. 1983. Biochemistry 22:4625-37

24. Cleland, W. W. 1975. Biochemistry 14:3220-24

25. Cleland, W. W. 1980. Methods Enzymol. 64:104-25

26. Bigeleisen, J. 1955. J. Chem. Phys. 23:2264-67

27. Ishida, T., Bigeleisen, J. 1976. J. Chem. Phys. 64:4775-89

28. Saunders, W. H. Jr. 1985. J. Am. Chem. Soc. 107:164-69

29. Huskey, W. P., Schowen, R. L. 1983. J. Am. Chem. Soc. 105:57046

30. Amin, M., Price, R. C., Saunders, W. H. Jr. 1988. J. Am. Chem. Soc. 110:40:35

31. Limbach, H.-H., Hennig, J., Gerritzen, D., Rumpel, H. 1982. Faraday Discuss. Chem. Soc. 74:229-43

32. Blanchard, J. S., Cleland, W. W. 1980. Biochemistry 19:3543-50

33. Hermes, J. D., Morrical, S. W., O'Leary, M. H., Cleland, W. W. 1984. Biochemistry 23:5479-88

34. Parkin, D. W., Schramm, V. L. 1987. Biochemistry 26:913-20

35. Hermes, J. D., Tipton, P. A., Fisher, M. A., O'Leary, M. H., Morrison, J. F., Cleland, W. W. 1984. Biochemistry 23:6263-75

36. Abell, L. M., O'Leary, M. H. 1988. Biochemistry 27:5933-39

37. Abell, L. M., O'Leary, M. H. 1988. Biochemistry 27:5927-33

38. Grissom, C. B., Cleland, W. W. 1985. Biochemistry 24:944-48

39. Grissom, C. B., Cleland, W. W. 1986. J. Am. Chem. Soc. 108:5582-83

40. Grissom, C. B., Willeford, K. O., Wedding, R. T. 1987. Biochemistry 26:2594-96

41. Uhr, M. L., Thompson, V. W., Cleland, W. W. 1974. J. Biol. Chem. 249:2920-27

42. Northrop, D. B., Cleland, W. W. 1974. J. Biol. Chem. 249:2928-31

43. O'Leary, M. H., Limburg, J. A. 1977. Biochemistry 16:1129-35

44. Grissom, C. B., Cleland, W. W. 1988. Biochemistry 27:2934-43

45. Rendina, A. R., Hermes, J. D., Cleland, W. W. 1984. Biochemistry 23:6257-62

46. Pascal, R. A. Jr., Walsh, C. T. 1984. Biochemistry 23:2745-52

47. Clark, J. D., O'Keefe, S. J., Knowles, J. R. 1988. Biochemistry 27:5961-71
48. Lenz, H., Eggerer, H. 1976. Eur. J. Biochem. 65:237-46

49. Miziorko, H. M., Lorimer, G. H. 1983. Annu. Rev. Biochem. 52:507-35

50. Van Dyk, D. E., Schloss, J. V. 1986. Biochemistry 25:5145-56

51. Roeske, C. A., O'Leary, M. H. 1984. Biochemistry 23:6275-84

52. Havir, E. A., Hanson, K. R. 1975. Biochemistry 14:1620-29

53. Hermes, J. D., Weiss, P. M., Cleland, W. W. 1985. Biochemistry 24:2959-67

54. Hermes, J. D., Cleland, W. W. 1984. J. Am. Chem. Soc. 106:7263-64

55. O'Keefe, S. J., Knowles, J. R. 1986. J. Am. Chem. Soc. 108:328-29

56. O’Keefe, S. J., Knowles, J. R. 1986. Biochemistry 25:6077-84

57. Albery, W. J., Knowles, J. R. 1986. Biochemistry 25:2572-77, and preceding papers in Biochemistry 25

58. Belasco, J. G., Bruice, T. W., Albery, W. J., Knowles, J. R. 1986. Biochemistry 25:2558-64

59. Albery, W. J., Knowles, J. R. 1987. J. Theor. Biol. 124:137-71

60. Albery, W. J., Knowles, J. R. 1987. J. Theor. Biol. 124:173-89

61. Klinman, J. P. 1981. CRC Crit. Rev. Biochem. 10:39-80

62. Welsh, K. M., Creighton, D. J., Klinman, J. P. 1980. Biochemistry 19:200516

63. Swain, C. G., Stivers, E. C., Reuwer, J. F. Jr., Schaad, L. J. 1958. J. Am. Chem. Soc. 80:5885-93

64. O'Leary, M. H., Yamada, H., Yapp, C. J. 1981. Biochemistry 20:1476-81

65. Marlier, J. F., O'Leary, M. H. 1986. J. Am. Chem. Soc. 108:4896-99

66. Abell, L. M., O'Leary, M. H. 1988. Biochemistry 27:3325-30

67. Weiss, P. M., Garcia, G. A., Kenyon, G. L., Cleland, W. W., Cook, P. F. 1988. Biochemistry 27:2197-205

68. Jordan, F., Kuo, D. J., Monse, E. U. 1978. J. Am. Chem. Soc. 100:287278

69. Weiss, P. M., Cook, P. F., Hermes, J. D., Cleland, W. W. 1987. Biochemistry 26:7378-84

70. Weiss, P. M., Chen, C.-Y., Cleland, W. W., Cook, P. F. 1988. Biochemistry 27:4814-22

71. Silverman, D. N., Vincent, S. H. 1984. CRC Crit. Rev. Biochem. 14:207-55

72. Paneth, P., O'Leary, M. H. 1987. Biochemistry 26:1728-31

73. Attwood, P. V., Tipton, P. A., Cleland, W. W. 1986. Biochemistry 25:8197-205

74. Chcung, Y.-F., Walsh, C. T. 1976. Biochemistry 15:3749-55 